\title{
Internal Transport Barrier Analysis Including Impurities in Tokamak and Helical Reactor Plasmas*)
}

\author{
Yoshihito HORI, Kozo YAMAZAKI, Tetsutarou OISHI, Hideki ARIMOTO and Tatsuo SHOJI \\ Nagoya University, Furo-cho, Chikusaku, Nagoya 464-8603, Japan
}

(Received 10 December 2010 / Accepted 21 April 2011)

\begin{abstract}
The operation with Internal Transport Barrier (ITB) is expected as a high performance operation. ITB is utilized to improve core plasma confinement in the reversed magnetic shear. It is considered that the changes of core plasma profile by the ITB cause changes of impurity transport. In a large fusion reactor, high- $Z$ materials will be used as plasma facing components because high loads of heat and particles concentrate there. However, high- $Z$ impurities from these components cause large radiation loss and dilute the fuel even if the amount of impurities is small. Therefore, in this study, firstly, the ITB formation which includes the effects of the magnetic shear and perturbed profiles by the pellet injection was simulated using the Toroidal Transport Analysis Linkage code TOTAL. Secondly, we analyzed transport of the tungsten impurities using an impurity model in TOTAL code, and compared the impurity profile in the case with ITB to the one without ITB in the tokamak reactor. The impurities decreased in the ITB formation region when ITB was formed, and the outward flux of total impurity density was observed there. It can be expected that outward flux of impurities is generated by the temperature and the density gradients.
\end{abstract}

(C) 2011 The Japan Society of Plasma Science and Nuclear Fusion Research

Keywords: transport simulation, internal transport barrier, impurity, pellet injection, tokamak reactor

DOI: $10.1585 /$ pfr.6.2403140

\section{Introduction}

In fusion reactors, it is difficult to control the temperature and the current distribution only by external heating and the current drive techniques, because the plasma is self-burning. Therefore, it is important to control externally the density profile of the core plasma to optimize reactor operations. For an operation, it is considered that an advanced operation scenario using reversed magnetic shear mode and higher poloidal beta mode with internal transport barrier (ITB) will be needed for confinement improvement and higher bootstrap current fraction in future reactors. It is expected that reliable operation with ITB enables an optimized operation to improve burning plasma confinement. However, in the reactor with a large fusion power output, the plasma facing components using high- $Z$ materials will be needed because high heat loads concentrate on these regions such as the divertor plates. The chemical and physical sputtering processes on these components yield impurities into the core plasma. The high- $Z$ impurity ions tend to accumulate in the plasma core due to inwardly directed drift velocities caused by neo-classical convection, cause large radiation loss and dilute the fuel even with a small amount of impurities.

The transport simulations have been carried out focusing on the ITB formation in tokamak and helical plasmas. The ITB model based on Bohm and GyroBohm-like trans-

author'se-mail: yamazaki@ees.nagoya-u.ac.jp

*) This article is based on the presentation at the 20th International Toki Conference (ITC20). port with $\boldsymbol{E} \times \boldsymbol{B}$ shear flow effects has already been compared with the JET experimental ITB in tokamak systems [1] and in helical system compared with LHD [2]. This model is introduced into the toroidal transport linkage TOTAL code $[3,4]$, and is applied to the 1-dimensional (1-D) ITB formation simulation of 2-D equilibrium for tokamk plasmas and 3-D equilibrium for helical plasmas.

Section 2 will describe the details of the core and impurity transport models and pellet injection model included in the TOTAL code, and simulation results will be shown in section 3. The conclusion will be given in section 4 .

\section{Numerical Model}

\subsection{Transport model description}

The Bohm and GyroBohm mixed transport model with the $\boldsymbol{E} \times \boldsymbol{B}$ shear flow effect has already been compared with the helical and tokamak experimental ITBs [1,2]. The most widely accepted explanation for the ITB formation relies on the suppression of ITG turbulence due to $\boldsymbol{E} \times \boldsymbol{B}$ shear flow. The suppression of the turbulence might occur when the $\boldsymbol{E} \times \boldsymbol{B}$ flow shearing rate $\omega_{\boldsymbol{E} \times \boldsymbol{B}}$ exceeds the ITG linear growth rate $\gamma_{\mathrm{ITG}}$. The shearing rate $\omega_{\boldsymbol{E} \times \boldsymbol{B}}$ is defined as $[5,6]$

$$
\omega_{\boldsymbol{E} \times \boldsymbol{B}} \cong\left|\frac{R B_{\theta}}{B_{\phi}} \frac{\partial}{\partial r}\left(\frac{E_{r}}{R B_{\theta}}\right)\right|,
$$

where $E_{r}, B_{\theta}$ and $B_{\phi}$ are the radial electric field, the poloidal and toroidal magnetic field, respectively. In tokamaks, the radial electric field $E_{r}$ is not easily measured di- 
rectly, so that $E_{r}$ can be calculated from the plasma radial force balance equation. The ITG growth rate $\gamma_{\mathrm{ITG}}$ is defined as [7]

$$
\gamma_{\mathrm{ITG}}=\frac{\left(\eta_{\mathrm{i}}-2 / 3\right)^{1 / 2}|s| c_{\mathrm{i}}}{q R}, \quad s=\frac{r}{q}\left(\frac{\mathrm{d} q}{\mathrm{~d} r}\right),
$$

where $\eta_{\mathrm{i}}=L_{n} / L_{T}, L_{n}=-\nabla n / n, L_{T}=-\nabla T_{\mathrm{i}} / T_{\mathrm{i}}, c_{\mathrm{i}}=$ $\sqrt{T_{\mathrm{i}} / m_{\mathrm{i}}}$, and $s$ is the magnetic shear.

Thermal diffusion coefficient $\chi$ is described in the form

$\chi_{\mathrm{e}, \mathrm{i}}=\chi_{\text {neoclassical }}+\chi_{\text {anomalous }}$,

$\chi_{\text {anomalous }}=\left(\alpha_{1} \times \chi_{\text {Gyrobohm }}+\alpha_{2} \times \chi_{\text {Bohm }}\right) \times F\left(\omega_{\boldsymbol{E} \times \boldsymbol{B}} / \gamma_{\mathrm{ITG}}\right)$,

where stabilization factor $F\left(\omega_{\boldsymbol{E} \times \boldsymbol{B}} / \gamma_{\mathrm{ITG}}\right)$ is defined using the ratio of $\omega_{\boldsymbol{E} \times \boldsymbol{B}}$ and $\gamma_{\mathrm{ITG}}$ as

$$
F\left(\omega_{E \times \boldsymbol{B}} / \gamma_{\mathrm{ITG}}\right)=\frac{1}{1+\left\{\tau \times\left(\omega_{\boldsymbol{E} \times \boldsymbol{B}} / \gamma_{\mathrm{ITG}}\right)\right\}^{\gamma}} .
$$

The coefficient $\chi_{\text {neoclassical }}$ is the neoclassical part of thermal diffusion coefficient, and $\chi$ anomalous is the anomalous part described as the Bohm and GyroBohm mixed transport model [8]. And particle diffusion coefficient $D$ is assumed as $D=\chi_{\mathrm{e}, \mathrm{i}} / C_{\mathrm{ano}}$, in this paper $C_{\mathrm{ano}}=3, \tau=2.0$, and $\gamma=4.0$ in tokamak, and $\tau=15.0$, and $\gamma=2.0$ in helical case. The parameters are determined by a comparison with the experimental data of JT-60U and LHD [8-10]. Two typical JT-60U parameters in Table 1 are shown in the positive magnetic shear case and the reversed case for ion temperature profile simulation.

\subsection{Pellet injection}

The pellet injection is described as the process of the pellet ablation. We used here the most popular one: the neutral gas shielding (NGS) model [11]. This ablation rate by the scaling is described as

$$
\frac{\mathrm{d} N}{\mathrm{~d} x}=1.12 \times 10^{16} n_{\mathrm{e}}^{0.333} T_{\mathrm{e}}^{1.64} r_{\mathrm{p}}^{1.333} M_{\mathrm{i}}^{-0.333} V_{\mathrm{p}}^{-1}[\text { atoms } / \mathrm{m}],
$$

where $n_{\mathrm{e}}, T_{\mathrm{e}}, r_{\mathrm{p}}, M_{\mathrm{i}}$ and $V_{\mathrm{p}}$ are electron density, electron temperature, pellet radius, particle mass in pellet and injection velocity, respectively.

\subsection{Impurity model}

We examined high- $Z$ impurities with a model for impurities in TOTAL; the multi-species dynamic impurity code IMPDYN [12] was used to model the ionization

Table 1 Typical machine and reactor parameters.

\begin{tabular}{cccccccc}
\hline Device & $\begin{array}{c}\text { Shot } \\
\text { number }\end{array}$ & $\begin{array}{c}R \text { maj } \\
{[\mathrm{m}]}\end{array}$ & $\begin{array}{c}a \min \\
{[\mathrm{m}]}\end{array}$ & $B \mathrm{t}[\mathrm{T}]$ & $\delta$ & $\kappa$ & Shear \\
\hline \hline JT-60U & $\# 21811^{*}$ & 3.424 & 0.944 & 4.008 & 0.185 & 1.43 & Positive \\
JT-60U & $\# 32423^{*}$ & 3.368 & 0.7949 & 3.836 & 0.2319 & 1.597 & Reversed \\
ITER & - & 6.20 & 2.00 & 5.30 & 0.33 & 1.70 & - \\
HR-1 & - & 16.80 & 2.60 & 4.21 & - & - & - \\
\hline
\end{tabular}

* from International Multi-Tokamak Confinement Profile states, and the NCLASS code [13] was used for the full neoclassical transport of each charge state considering arbitrary aspect ratio and collisionality.

For the impurity dynamics $[14,15]$, the rate equation and transport equation are solved using IMPDYN code [12] coupled with the ADPAK atomic physics package [16], which are described as

$$
\begin{aligned}
\frac{\partial n_{k}}{\partial t} & =-\frac{1}{V^{\prime}} \frac{\partial}{\partial \rho}\left(V^{\prime} \Gamma_{k}\right) \\
& +\left[\gamma_{k-1} n_{k-1}+\alpha_{k+1} n_{k+1}-\left(\gamma_{k}+\alpha_{k}\right) n_{k}\right] n_{\mathrm{e}}+S_{k}, \\
\Gamma_{k}= & \Gamma_{k}^{\mathrm{NC} s}+\Gamma_{k}^{\mathrm{NC} a}-D_{k}(\rho) \frac{\partial n_{k}}{\partial \rho}+V_{k}(\rho) n_{k},
\end{aligned}
$$

with ionization rate $\gamma_{k}$, recombination rate $\alpha_{k}$, and particle source term $S_{k}$. Here, a constant anomalous diffusion coefficient $D_{k}$ and simply modeled inward velocity $V_{k}=V(a)(r / a)$ are used for impurity anomalous transport ( $V_{k}<0$ corresponds to inward velocity). The main fuel neutrals are calculated by the AURORA Monte Carlo code [17].

The neoclassical impurity flux in tokamak is expressed by

$$
\begin{aligned}
& \Gamma_{k}^{\mathrm{NC} s}=-D_{k}^{\mathrm{NC}} \nabla n_{k} \\
& \quad+D_{k}^{\mathrm{NC}} n_{k}\left[\sum_{l \neq k}\left(g_{n l \rightarrow k} \nabla n_{l} / n_{l}\right)+g_{T_{\mathrm{i}}} \nabla T_{\mathrm{i}} / T_{\mathrm{i}}+g_{T_{\mathrm{e}}} \nabla T_{\mathrm{e}} / T_{\mathrm{e}}\right],
\end{aligned}
$$

where several coefficients $g$ are calculated using the NCLASS code.

In the simulation, the impurity source was defined as the impurity neutral flux on the plasma boundary. The neutral impurity density profile $n_{0}(\rho)$ is assumed to be

$$
n_{0}(\rho)=-\frac{V^{\prime}(1) \Gamma_{0}(1)}{V^{\prime}(\rho) v_{0}} \exp \left(-\frac{1}{v_{0}} \int_{1}^{\rho} \mathrm{d} \rho n_{\mathrm{e}}(\rho) \gamma_{0}(\rho)\right),
$$

derived from

$$
\begin{aligned}
& \frac{\partial n_{0}}{\partial t}=-\frac{1}{V^{\prime}} \frac{\partial}{\partial \rho}\left(V^{\prime} \Gamma_{0}\right)-\gamma_{0} n_{\mathrm{e}} n_{0} \approx 0, \\
& \Gamma_{0}(\rho) \approx-n_{0}(\rho) v_{0} .
\end{aligned}
$$

Here, $\Gamma_{0}(1)$ is the neutral impurity flux at the plasma boundary $(\rho=1)$, and $v_{0}$ is the neutral impurity inward velocity (assuming an energy of $10 \mathrm{eV}$ ). The symbols $\gamma_{0}$ and $n_{\mathrm{e}}$ are the ionization coefficient and electron density near the plasma boundary, respectively.

To clarify the effect of impurity ions, stationary state burning plasma condition were established without impurities. Then, a continuous neutral impurity influx was introduced, and after a transient phase, the system settled into a new radiation-enhanced stationary state.

\section{Simulation Results 3.1 The ITB formation}

The pellet injection makes the local density gradient quickly, and the electric field gradient is formed. Because 
the shear flow $\omega_{\boldsymbol{E} \times \boldsymbol{B}}$ grows in the reversed shear region, the stabilization requirement $\omega_{\boldsymbol{E} \times \boldsymbol{B}}>\gamma_{\mathrm{ITG}}$ can be satisfied, which leads to the reduction of transport coefficients.

Figure 1 shows the radial profiles of the stationary state in these operations with pellet injection (penetration length: $r / a \sim 0.4$ ). In Fig. 1, (a) shows the positive magnetic shear case without ITB, and (b) is the reversed case with ITB. The electron temperature, density and thermal diffusion coefficient are compared.

ITB is formed in the reversed magnetic shear, however it is not formed in the positive magnetic shear. It is considered that the ITB is formed easily in the reversed magnetic shear because the instability term (ITG linear growth rate) becomes small on the condition that the magnetic shear becomes small and stabilization occurs in this model. The pellet injection from the outer midplane also contributes this stabilization, because it causes the density gradient and the electric field gradient locally in the core plasma. This large electric field gradient increases the stable term $\omega_{\boldsymbol{E} \times \boldsymbol{B}}$. Therefore, it is considered that the control of ITB formation by the external actuator such as the pellet injection is important.

In the present model, the positive or negative q-profile is a priori assumed. Actually the external current drive and the bootstrap current profiles should be considered selfconsistently. We neglect these issues in the present simulation. It should be also noted that the pellet injection is assumed as a continuous fueling model, not a discrete model.

In the helical case, HR-1 parameters in Table 1 are used. HR-1 reactor is designed based on LHD by the Physics-Engineering-Cost (PEC) code [18], which has $1 \mathrm{GW}$ electric power. Figure 2 shows the radial profiles of this simulation result with pellet injection (penetration
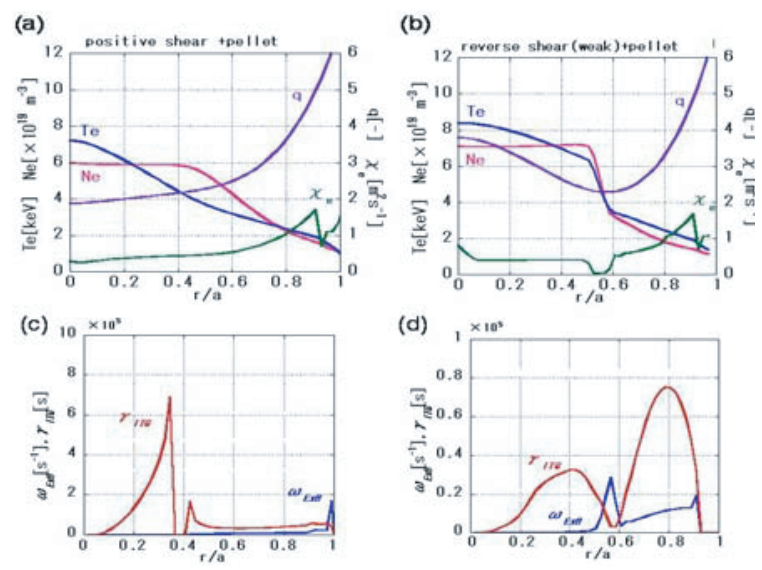

Fig. 1 The radial profiles (safety factor, electron density and temperature, thermal diffusion coefficient) in tokamak case with pellet injection. Left figures (a) and (c) denote the positive shear case, and right figures (b) and (d) are the reversed shear case. The figures (a) and (b) show radial profiles of safety factor, electron temperature, density and thermal diffusion coefficient. Figures (c), (d) show profiles of $\omega_{E \times \boldsymbol{B}}$ and $\gamma_{\mathrm{ITG}}$. length: $r / a \sim 0.2)$. The ITB formation was also confirmed in a helical reactor.

\subsection{Transport analysis of impurities in the presence of ITB}

Impurities (tungsten) were added, and the transport analysis of impurities was done using the ITER parameter shown in the Table 1. For core plasma simulation in this analysis, the thermal diffusion coefficient of plasma was based on the model fitted with JT-60U experimental results as shown in Section 2.1. Here, impurity ion temperature is assumed to be the same as bulk ion temperature. Tungsten impurities were given as the neutral fluxes $10^{18} \mathrm{~m}^{-2} \mathrm{~s}^{-1}$ from the separatrix.

Figure 3 shows the time evolution of the fusion power, heating power and average electron temperature in this operation. Impurities were added at $100 \mathrm{~s}$ in the stationary state. Then, the outputs decrease temporarily, and they become stationary state again. This stationary state is discussed in this section.

Figure 4 shows the radial profiles of the core plasma and tungsten impurity density at the $160 \mathrm{~s}$ in Fig. 3. The core plasma profiles (electron density, temperature and thermal diffusion coefficient) are shown in (a) and (b), the tungsten density profiles of each ion and their summation denoted as "total" in this figures are shown in (c) and (d). Left figures: (a) and (c) are the cases of operation without ITB, and right figures are the cases with ITB. In the present model, the ITB formation is determined roughly by
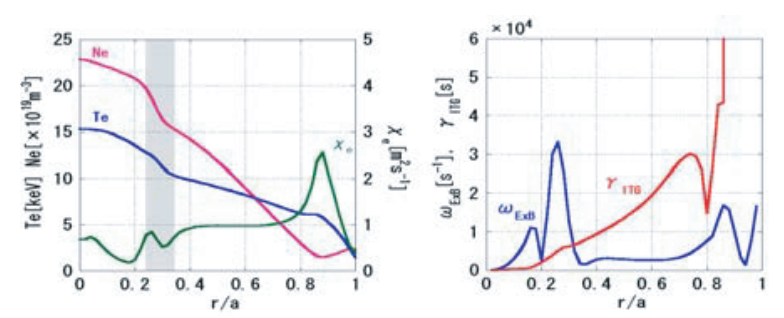

Fig. 2 The radial profiles in the stationary state in helical case. Left figure shows profiles of radial electric field, electron density, temperature and thermal diffusion.

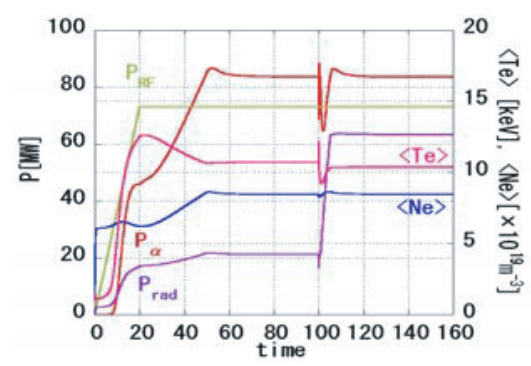

Fig. 3 ITER plasma simulation with impurities added at $100 \mathrm{~s}$, showing time-evolutions of alpha heating power $P_{\mathrm{a}}$, external heating power $P_{\mathrm{RF}}$, radiation power $P_{\text {rad }}$, average electron temperature $\left\langle T_{\mathrm{e}}\right\rangle$ and average electron density $\left\langle N_{\mathrm{e}}\right\rangle$. 

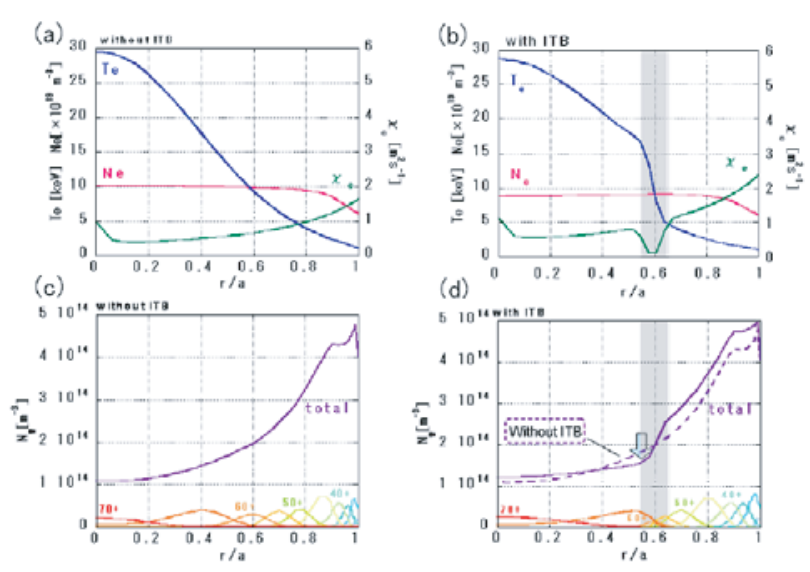

Fig. 4 The radial profiles at $160 \mathrm{~s}$. Above figures show electron density $N_{\mathrm{e}}$, temperature $T_{\mathrm{e}}$ and thermal diffusion coefficient $\chi_{\mathrm{e}}$ profiles. Below figures show the tungsten density profiles of each ion and their summation (denoted as "total" in this figures). Left figures show without ITB formation cases. Right figures are with ITB formation cases.

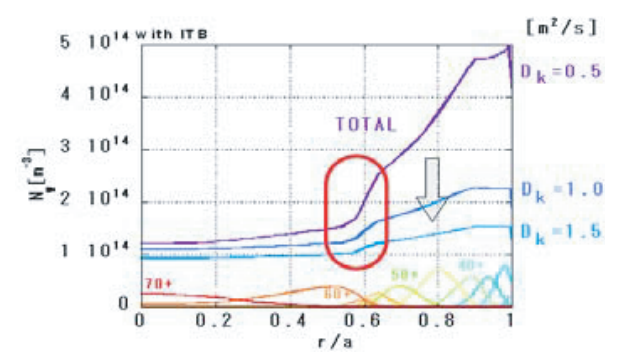

Fig. 5 The impurity profiles in the anomalous transport term: $D_{k}=0.5,1.0,1.5 \mathrm{~m}^{2} / \mathrm{s}$ case in the operation with ITB.

$\omega_{\boldsymbol{E} \times \boldsymbol{B}}>\gamma_{\mathrm{ITG}}$. The time-evolution of the criterion parameter depends on magnetic shear and local density gradient induced by the pellet injection. Here we assumed typical core transport coefficient without or with ITB to clarify impurity ion dynamics.

In these results, the change in the radial profile of high $Z$ impurities (tungsten) was observed in the presence of ITB. The impurities decreased in the ITB region when ITB was formed, and the outward flux of total impurity density was observed there (but, each-Z-state impurity has inward or outward flux so that their summation is outward). It is considered that this caused by thermal and density gradients of the back ground plasma. These gradients cause the decrease of inward velocity and the change of the tungsten impurity density profile in the ITB region, because the particle fluxes based on the neoclassical theory in the transport equation depend on the temperature and density gradients in this impurity transport model.

In this ITER simulation, the impurity radiation effect is not so serious, because the radiation loss effect is localized to the outer region and the central plasma is sustained by alpha and external heating powers. However, the accumulation of impurities was observed in the center in both cases, which is significant problem in the future reactor.

Figure 5 shows the result in the case the anomalous term in the impurity transport equation is changed. In this simulation, the neoclassical diffusion coefficient was about $0.5 \mathrm{~m}^{2} \mathrm{~s}^{-1}$ so that we gave $D_{k} \sim 0.5,1.0,1.5 \mathrm{~m}^{2} \mathrm{~s}^{-1}$ uniformly as the anomalous term, respectively. When anomalous term was large, impurity profiles was close to flat, and the change in the ITB region become small. It is because the neoclassical diffusion term was small and the anomalous term did not depend on the thermal and density gradients. In these cases, the accumulation of impurities in the center was also observed.

\section{Conclusion}

The effect of the magnetic shear and pellet injection on the ITB formation was simulated. The utility of the ITB formation with the density control by the pellet injection was shown. Then, tungsten impurity transport was analyzed in the presence of ITB. The change of impurity transport was observed in the presence of ITB, and the decrease of impurities could be confirmed in the ITB formation region. However, the significant change did not appear in the impurity density accumulated in the center. In the case that ITB is formed, it can be expected that outward flux of impurities is generated by the temperature and the density gradient.

The impurity transport in a helical reactor is under analysis now.

[1] T. Tala et al., Plasma Phys. Control. Fusion 43, 507 (2001).

[2] J. Garcia, K. Yamazaki, J. Dies and J. Izquierdo, Phys. Rev. Lett. 96, 105007 (2006).

[3] K. Yamazaki and T. Amano, Nucl. Fusion 32, 633 (1992).

[4] K. Yamazaki et al., Fusion Eng. Des. 81, 2743 (2006).

[5] T.S. Hahm and K.H. Burrell, Phys. Plasmas 2, 1648 (1995).

[6] P. Zhou, W. Horton and H. Sugama, Phys. Plasmas 6, 2503 (1999).

[7] B. Esposito et al., Plasma Phys. Control. Fusion 45, 933 (2003).

[8] Y. Hori et al., Plasma Fusion Res. 5, S2034 (2010).

[9] T. Fujita et al., Phys. Rev. Lett. 78, 2377 (1997).

[10] T. Shimozuma et al., Plasma Phys. Control. Fusion 45, 1183 (2003).

[11] P.B. Parks and R.J. Turnbull, Phys. Fluids 21, 1735 (1978).

[12] T. Amano, J. Mizuno and J. Kako, Internal report IPPJ-616, Institute of Plasmas Physics, Nagoya Univ. (1982).

[13] W.A. Houlberg et al., Phys. Plasmas 4, 3230 (1997).

[14] Y. Murakami, T. Amano et al., J. Nucl. Mater. 313-316, 1161 (2003).

[15] K. Yamazaki et al., J. Plasma Fusion Res. SERIES 7, 102 (2006).

[16] R.A. Hulse, Nucl. Technol. Fusion 3, 259 (1983).

[17] M.H. Huges and D.E. Post, J. Comput. Phys. 28, 43 (1978).

[18] K. Yamazaki et al., Fusion Eng. Des. 81, 2743 (2006). 